\title{
Barotropic mode errors in an Indian Ocean model associated with the GWR method
}

\author{
Tommy G. Jensen* \\ School of Ocean and Earth Science and Technology, International Pacific Research Center, University of Hawai'i at Manoa, \\ 1680 East West Rd. POST Bldg. 413F Honolulu, HI 96822, USA
}

Received 30 March 2001; accepted 6 July 2002

\begin{abstract}
Fast barotropic gravity waves in the ocean require that an efficient computational method is applied in ocean models used for climate simulations. The gravity wave retardation (GWR) method is a simple technique that slows down surface waves and allows a simple explicit integration to be used. Here the method is discussed and applied to a layer model of the tropical Indian Ocean subject to monthly climatological wind forcing. The errors introduced by the GWR method on the barotropic ocean circulation and sea surface elevation are analyzed. Comparison to a model integration with a flat bottom demonstrates that GWR integrations with a speed-up factor up to 16 indeed capture some influence of the bottom relief, in the sense that the GWR solutions have less error than if topography was ignored. Integrations with a speed-up factor of up to 8 are found to model the barotropic circulation well, implying that the GWR method can be applied to climate modelling.
\end{abstract}

(C) 2003 Elsevier Science B.V. All rights reserved.

Keywords: Barotropic ocean circulation; Gravity wave retardation; Indian Ocean

\section{Introduction}

State of the art climate modelling requires long integrations of ocean models with relatively high resoluion. This demands very powerful computing resources, most easily provided by massively parallel computers. To take advantage of these resources, a code must be very close to $100 \%$ parallelized, which limits our choice of suitable algorithms. When based on the equations of an incompressible fluid, the fastest propagating waves in an ocean general circulation model (OGCM) are long gravity waves. They

* Tel.: +1-808-956-5468; fax: +1-808-956-9425.

E-mail address: jensen@soest.hawaii.edu (T.G. Jensen). propagate with phase speeds exceeding internal modes by two orders of magnitude, and provide adjustment to geostrophically balanced flow at a time scale much shorter than those of interest in climate modelling.

Oberhuber (1993) applied a semi-implicit method to an isopycnal OGCM for all layers, but more commonly, a separation into barotropic and baroclinic modes is made, so that two different algorithms can be applied. The slow baroclinic mode is usually solved by an explicit time integration scheme. For the fast mode, an elliptic equation can be formulated by introducing a streamfunction (e.g. Bryan, 1969; Semtner, 1986), or by treating the surface elevation implicitly (Dukowicz and Smith, 1994). With this method, the parallelization problem 
is primarily associated with the barotropic mode. Many recent models solve the barotropic mode explicitly by sub-cycling, i.e. using a much smaller time step for the fast mode to ensure numerical stability (e.g. Blumberg and Mellor, 1987; Killworth et al., 1991; Bleck and Smith, 1990; Hallberg, 1997). In that case, parallelization is easy, but the solution of the barotropic mode is relatively costly. Bryan (1984) used accelerated physics to reach a steady solution for climate models, but this method distorts all waves and can only be applied for a spin-up of the ocean to a steady state.

The gravity wave retardation (GWR) method has been used and analyzed for layer models in simple basin geometries and for simple forcing fields (Jensen, 1996; Tobis, 1996). More recently, Jensen (2001) applied the GWR method successfully to a realistic ocean basin with observed wind forcing and demonstrated its relatively high accuracy and high efficiency. However, in Jensen $(1996,2001)$, the GWR method was mainly considered an alternative to reduced gravity models by including effects of bottom topography on the baroclinic modes. Consequently, the error analysis in those studies focused on the baroclinic modes.

In this paper, we will use the same model as Jensen (2001), but investigate the barotropic response. This is of particular interest for OGCMs for climate modelling, since the GWR method easily can be applied to a sub-cycled barotropic mode to save significant computer time. In fact, Hearn and Hunter (1987) and Hunter (1990a,b) used the equivalent of this method to spin-up barotropic ocean models on an $f$-plane to investigate flows in coastal regions subject to steady wind forcing.

\section{The GWR method}

The method works by slowing down the phase speeds of the barotropic gravity waves. Hearn and Hunter (1987) simply reduced the gravitational constant since they applied it to a homogeneous sea, while Jensen (1996) extended the method to the stratified case and demonstrated the analogy of the GWR method to the method of artificial compressibility (Chorin, 1967) by introducing a simple modification of the continuity equation. In a layer model, this results in a multiplication factor $\gamma$ to the surface elevation $\eta$ in the pressure gradient term:

$\nabla p_{j}=g\left(\gamma \rho_{j} \nabla \eta-\sum_{i=1}^{j-1}\left[\left(\rho_{j}-\rho_{i}\right) \nabla H_{i}\right]\right)$,

where $g$ is the constant gravitational acceleration, $\rho_{j}$ is the density, and $H_{j}$ is the thickness of layer $j$. Phase speeds of barotropic gravity waves are changed by a factor of the square root of $\gamma$, allowing an increase in time step by a factor of $\Gamma=\sqrt{1 / \gamma}$ for an explicit integration. Details of the derivation are given in Jensen (1996). We will refer to $\gamma$ as the GWR parameter and to $\Gamma$ as the GWR speed-up factor.

An appealing interpretation of how the method works was suggested by Cushman-Roisin (personal communication, 1996). By increasing the air density above the ocean, the effective gravity at the air-sea interface is only a fraction of its physical value. This affects all waves, but primarily ocean surface waves, which become internal waves in the modified atmosphere-ocean system. The equivalence of the GWR method to this modified physical system is only exact for a homogeneous ocean, but is closely related in the stratified case as shown below.

\section{Pressure gradient in a layered fluid}

Consider a fluid with $N+1$ isopycnal layers in the vertical and a free surface $\eta(\phi, \theta)$ and the bottom at $-D(\phi, \theta)$, where $\phi$ is the longitude and $\theta$ is the latitude (Fig. 1). The hydrostatic pressure in the upper layer (layer 0 ) is given by:

$p_{0}(z)=-g \rho_{0} z$

where the height $z$ of a density surface is negative in the fluid and zero at the surface $\eta$. A deeper layer $j$ has its upper boundary at:

$z_{T j}=-\sum_{i=0}^{j-1} H_{i}$

so we find:

$p_{j}(z)=p_{j-1}\left(z_{T j}\right)-g \rho_{j}\left(z-z_{T j}\right)$ 


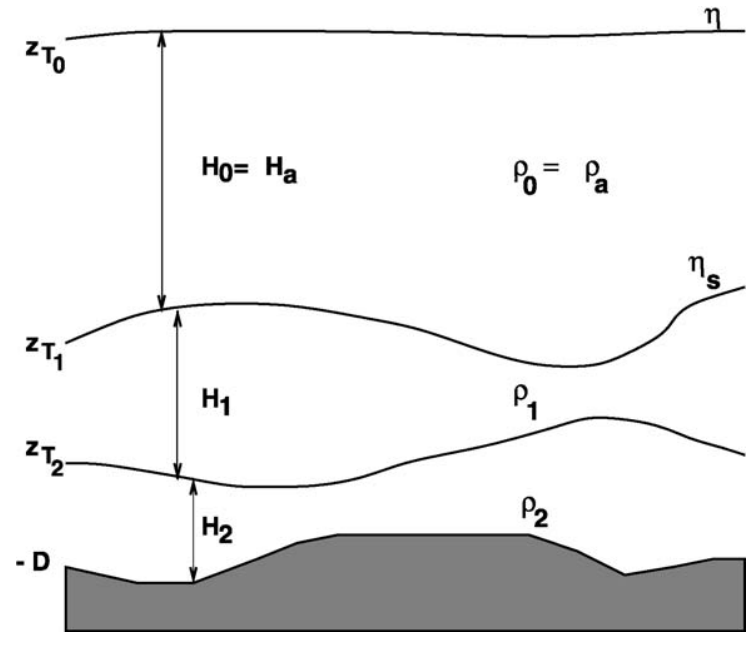

Fig. 1. A general layer model with a free surface and bottom topography confined to the deepest layer.

where the pressure at the top of layer $j$ equals the pressure at the bottom of layer $j-1$, e.g.:

$p_{j-1}\left(z_{T j}\right)=\sum_{i=0}^{j-1} g \rho_{i} H_{i}$.

We need to find the horizontal pressure gradient, vertically integrated over each layer. In a $z$-coordinate system, it is straightforward to integrate first and then use the Leibnitz formula to obtain this quantity. However, if we keep in mind that the density coordinate varies in the horizontal direction so that:

$\nabla z=-\nabla \eta=-\nabla \sum_{i=0}^{N}\left(H_{i}-D\right)$

where the gradient is along constant density surfaces, we find, taking the gradient of Eq. (4) and inserting the definitions of Eqs. (3) and (5):

$\nabla p_{j}=g\left[\rho_{j} \nabla \eta-\nabla \sum_{i=0}^{j-1}\left(\rho_{j}-\rho_{i}\right) H_{i}\right]$.

\section{Modified atmosphere-ocean system}

Applying Eq. (7) to a system with an atmosphere with density $\rho_{0}=\rho_{\mathrm{a}}$ and thickness $H_{0}=H_{\mathrm{a}}$ over a two- layer ocean with densities $\rho_{1}$ and $\rho_{2}$ and layer thicknesses $H_{1}$ and $H_{2}$, respectively, we find for the atmosphere:

$\nabla p_{\mathrm{a}}=g \rho_{\mathrm{a}} \nabla \eta$,

for the upper ocean:

$\nabla p_{1}=g\left[\rho_{1} \nabla \eta-\left(\rho_{1}-\rho_{\mathrm{a}}\right) \nabla H_{\mathrm{a}}\right]$

and for the deeper ocean:

$\nabla p_{2}=g\left[\rho_{2} \nabla \eta-\left(\rho_{2}-\rho_{\mathrm{a}}\right) \nabla H_{\mathrm{a}}-\left(\rho_{2}-\rho_{1}\right) \nabla H_{1}\right]$.

The gradient of the elevation at the top of the atmosphere is given by:

$\nabla \eta=\nabla\left(H_{a}+H_{1}+H_{2}-D\right)=\nabla H_{\mathrm{a}}+\nabla \eta_{\mathrm{s}}$

where we have defined a sea surface elevation $\eta_{\mathrm{s}}$, assuming that the topography is confined to the ocean. Assuming a rigid lid $(\eta=0)$ on the top of the atmosphere gives:

$\nabla H_{\mathrm{a}}=-\nabla \eta_{\mathrm{s}}$

and the equations for the ocean become for layers 1 and 2 , respectively:

$\nabla p_{1}=g\left(\rho_{1}-\rho_{\mathrm{a}}\right) \nabla \eta_{\mathrm{s}}$

$\nabla p_{2}=g\left(\rho_{2}-\rho_{\mathrm{a}}\right) \nabla \eta_{\mathrm{s}}-g\left(\rho_{2}-\rho_{1}\right) \nabla H_{1}$.

For an unmodified atmosphere-ocean system, we have $\rho_{\mathrm{a}} \ll \rho_{1}$, and Eqs. (13) and (14) are given by Eq. (1) with $\gamma=1$. We also note that using $\gamma=\left(\rho_{\mathrm{o}}-\rho_{\mathrm{a}}^{*}\right) / \rho_{\mathrm{o}}$ in Eq. (1), where $\rho_{\mathrm{o}}$ is a reference density for seawater and $\rho_{\mathrm{a}}^{*}$ is an artificially increased air density is closely related, but not identical, to increasing the air density. The latter corresponds to choosing a slightly different $\gamma$ for each layer in Eq. (1), i.e.:

$\gamma_{j}=\left(\rho_{j}-\rho_{\mathrm{a}}^{*}\right) / \rho_{j}$.

\section{Relation to reduced gravity models}

Decreasing $\gamma$ in GWR models reduces the effective gravity in a way similar to reduced gravity models: in 
Eq. (1), the gradient of the surface elevation can be written as the gradient of the sum of all layer thicknesses, i.e.:

$$
\nabla \eta=\sum_{i=1}^{N} \nabla H_{i} .
$$

Reduced gravity models have a surface gradient forcing term given by:

$$
\nabla \eta=\sum_{i=1}^{N-1}\left(\frac{\rho_{N}-\rho_{i}}{\rho_{N}}\right) \nabla H_{i},
$$

(Jensen, 1991, 1996). The latter is equivalent to using a depth-varying value of $\gamma$ that is proportional to the density difference between each layer and the deepest layer. Substituting Eq. (17) for $\gamma \nabla \eta$ in Eq. (1) it is seen that the pressure gradient vanishes in the deepest layer, $N$, and the pressure gradients in the layers above are identical to those in a reduced gravity model. A reduced model is therefore a special case of a GWR model.

\section{Range of values for the GWR parameter $\gamma$}

It was shown in Jensen (1996), using the linear system of equations, that the equivalent depths for the modified system are found as eigenvalues to the matrix:

$a_{j i}=\left[\gamma-1+\frac{\rho_{\min (j, i)}}{\rho_{j}}\right] H_{0 j}$,

and the eigenvectors give the vertical structure of the flow. The subscript 0 indicates that a basic state at rest is used for this calculation. While the eigenvalues for the general case must found by a numerical solution, we can illustrate how the method works for the twolayer ocean with a density difference of $\Delta \rho$ between the lower and upper layers. If we chose to write the two-layer system as $H_{01}=\alpha H$ and $H_{02}=(1-\alpha) H$, where $0<\alpha<1$, we find from Eq. (18) that the two eigenvalues are:

$h^{(0,1)}=\frac{1}{2} \gamma H(1 \pm \sqrt{1-b})$, where:

$b=\frac{4 \Delta \rho \alpha(1-\alpha)}{\rho \gamma}$,

and the phase speeds are given by:

$c^{(0,1)}=\sqrt{g h^{(0,1)}}$.

Here the superscripts 0 and 1 refer to the barotropic mode and the baroclinic mode, respectively. For propagating solutions, the eigenvalues must be real, that is the term under the square root must be positive, i.e. $b \leq 1$. In the case of $b \ll 1$, we can expand the equivalent depths in Taylor series as:

$h^{0} \approx \gamma H\left(1-1 / 4 b-1 / 16 b^{2}\right)+O\left(b^{3}\right)$

and

$h^{1} \approx \frac{\Delta \rho \alpha(1-\alpha)}{\rho}\left(1+1 / 4 b+1 / 8 b^{2}+O\left(b^{3}\right)\right)$.

We note that to first order the phase speed of the barotropic mode is changed by a factor of $\sqrt{\gamma}$, while the baroclinic mode is unchanged. The higher order terms imply that the baroclinic mode in a GWR solution will have a slightly increased phase speed compared to the $\gamma=1$ case.

A special case is $\gamma=\Delta \rho / \rho$, where Eq. (19) can be solved exactly:

$h^{0}=\frac{\Delta \rho}{\rho}(1-\alpha) H ; \quad h^{1}=\frac{\Delta \rho}{\rho} \alpha H$.

One phase speed, $c^{0}$, becomes identical to that of a bottom boundary layer in an infinitely deep ocean, while the phase speed of the other mode becomes the same as found for a finite upper layer over an infinitely deep ocean. As far as phase speed is concerned, wave propagation has been replaced by two independent (baroclinic) reduced gravity wave solutions.

In the general case, the GWR parameter must be chosen sufficiently large that the barotropic mode remains significantly faster than the baroclinic modes, and so that all eigenvalues remain real. Using the two-layer case above as guidance, we find that in practice, $\gamma>\Delta \rho_{\max } / \rho$, where $\Delta \rho_{\max }$ is the maximum potential density difference in the open ocean, can be 
used to determine the maximum possible speed-up factor while maintaining physical meaningful solutions and numerical stability. Since this density difference is of order $10 \mathrm{~kg} / \mathrm{m}^{3}$, we can expect stability for a speed-up of an order of magnitude. Shallow surface layers of relative fresh water can be ignored since they do not influence the phase speed of the lowest order baroclinic mode. However, if a model explicitly includes shallow estuaries, where the thickness of fresh water outflow is a significant fraction of the total depth and assuming a density difference of about $25 \mathrm{~kg} / \mathrm{m}^{3}$, the speed-up factor is limited to a factor of $5-6$.

\section{Impact on barotropic wave propagation}

The effect of the method on the phase propagation of baroclinic gravity waves has been shown to be small, about $3-5 \%$ for a speed-up factor $\Gamma$ of 10 , and the effect on baroclinic Rossby waves is even smaller (Tobis, 1996; Jensen, 1996, 2001). Therefore, only the effect of the barotropic modes will be discussed here.

Since the GWR method works by slowing down barotropic gravity waves by an the order of magnitude, it obviously cannot be used to model any process that requires a good representation of their phase and energy propagation. As the phase speed is reduced, the barotropic Rossby radius of deformation is decreased by the same factor. As a result, geostrophic adjustment takes place over a much smaller area with very large surface displacements, and the increased deformation of the fluid column causes errors in the solution. The interpretation of surface waves as internal waves in a modified atmosphereocean system also help our understanding of the large surface elevations associated with the method. However, since $\gamma$ and $\eta$ appear only as a product in Eq. (1), the pressure gradients are fairly accurate, and the exaggerated surface elevation $\eta$ from a GWR solution can simply be rescaled by multiplication of $\gamma$ to obtain realistic values (Hearn and Hunter, 1987).

Barotropic Rossby waves are dispersive and the relative error associated with the GWR method depends on the wave number. Fig. 2 shows the ratio of the phase speed in a GWR model to the unmodified phase speed as function of the wavelength $\lambda$, scaled in

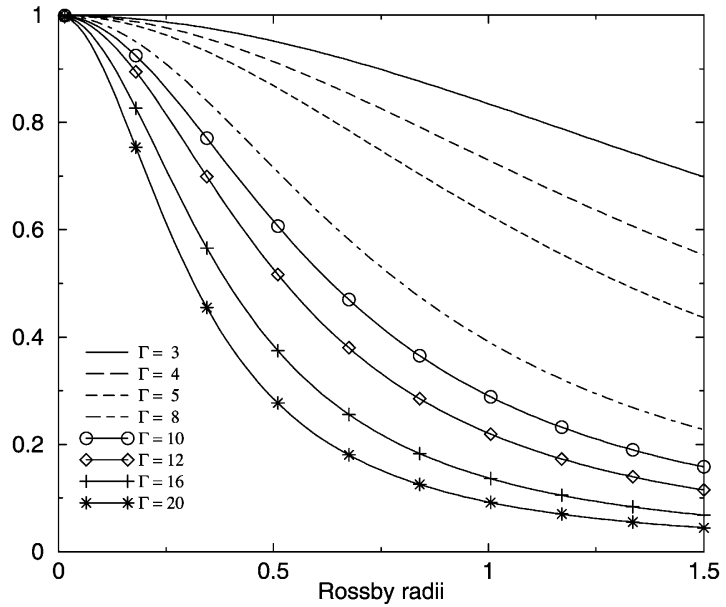

Fig. 2. Ratio of the phase speed of the barotropic Rossby waves in a GWR solution to the unmodified phase speed as function of wavelength in units of the (unmodified) Rossby radius of deformation. The same curves apply to the ratio of zonal group velocity components for barotropic Rossby waves with zero zonal wave number. Curves are shown for different values of the speed-up factor $\Gamma$.

units of the Rossby radius of deformation $a$ for $\Gamma=1$. The ratio of kinetic energy density to potential energy density is $(k a)^{2}$, where $k$ is the wave number (e.g. Gill, 1982 , p. 502), so Rossby waves with $k a \gg 1$ (or $\lambda \ll 2 \pi$ in Fig. 2) are associated with fast, horizontal motion. Waves with $k a \ll 1$ mainly represent changes in potential energy, and are not represented in Fig. 2. Recall the barotropic deformation radius is about 2000 $\mathrm{km}$ in the open ocean, so $k a=1$ corresponds to a wavelength of approximately the width of the Pacific Ocean. For $k a>2 \pi$, the barotropic wavelength is shorter that the external Rossby deformation radius. Fortunately, most barotropic waves, although the zonal scale may be basin-wide, are generated by weather systems with a meridional length scale of $1000 \mathrm{~km}$ or less, keeping $\mathrm{ka}$ much larger than 1 .

The group velocity of barotropic Rossby waves is more severely affected. Fig. 3 shows the ratio of the group velocity in the zonal direction for waves in a GWR model to the exact group velocity in the same direction as a function of the zonal wavelength scaled as in Fig. 2. As the speed-up factor $\Gamma$ is increased, the wavelength of the stationary Rossby wave $(k a=1)$ decreases and the range of wavelengths with eastward energy propagation is reduced. This means that bar- 


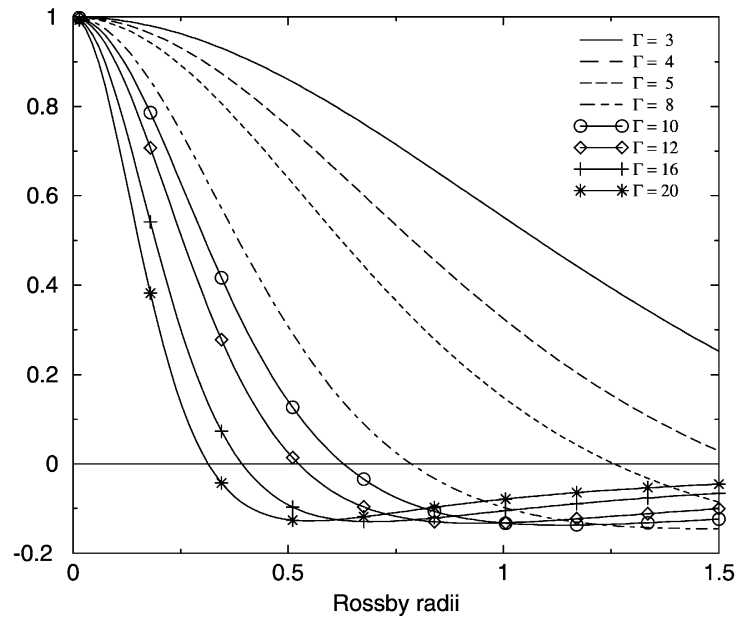

Fig. 3. As in Fig. 2, but for the group velocity in the zonal direction of barotropic Rossby waves with zero meridional wave number.

otropic waves in the open ocean, which should have eastward energy propagation if $k a<1$, instead will propagate energy westward if the GWR speed-up factor is sufficiently large. From Fig. 3, we note that waves longer than $1500 \mathrm{~km} \mathrm{(3/4}$ of the deformation radius) will propagate energy westward if $\Gamma$ exceeds
8. Fig. 3 was computed assuming that the meridional wave number is zero, but usually the zonal wave number is much smaller than the meridional wave number. If the zonal wave number is zero, the ratio of zonal group velocities is identical to the ratio of phase velocities, i.e. Fig. 2. However, as indicated by Fig. 3, the relative error may be still be large for waves that have a group velocity close to zero. The group velocity in the meridional direction is also affected. Fig. 2 can be used to obtain the ratio, since it is the square of the ratio of the phase speeds.

The shortest period of Rossby waves varies with latitude as

$T_{\max }=\frac{4 \pi R}{c} \tan (\phi)$

where $R$ is the radius of the Earth and $c$ the gravity phase speed. Barotropic Rossby waves with monthly periods can occur everywhere except within a few degrees of the north pole. However, as the phase speed is modified by the GWR method, the area where barotropic Rossby waves with a given frequency can exist decreases. For a speed-up factor of 5,10 , and 16 , respectively, the latitude decreases to $55^{\circ}, 35^{\circ}$, and $25^{\circ}$, respectively. For a bi-monthly

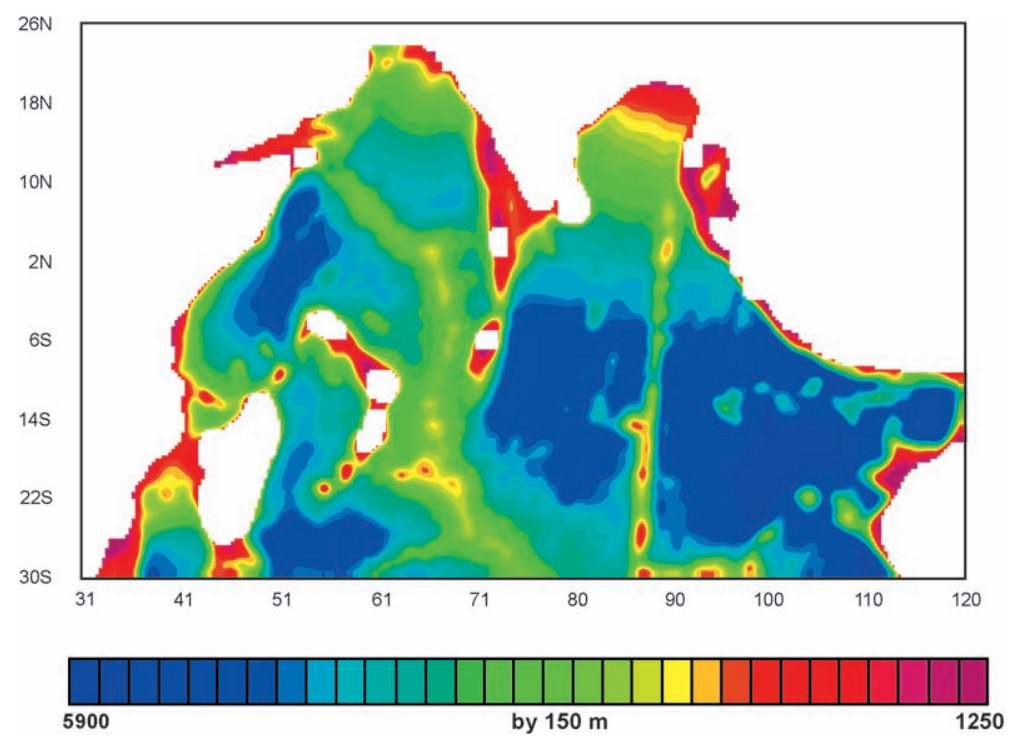

Fig. 4. Bottom topography for the Indian Ocean model. The minimum depth is $1200 \mathrm{~m}$, the maximum depth is $6000 \mathrm{~m}$, and the contour interval is $150 \mathrm{~m}$. 


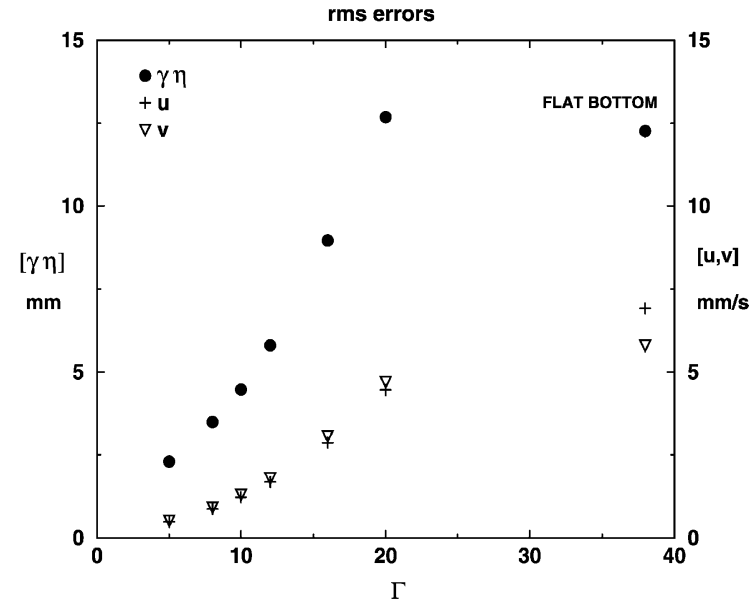

Fig. 5. Annual mean of the basin-wide root mean square (rms) error for the rescaled surface elevation, $\gamma \eta$ (circles), depth average of the zonal velocity component $u$ (plus) and the depth average of the meridional velocity component $v$ (triangle) as a function of the speed-up factor $\Gamma$. The label flat bottom indicates the rms error of the case of a flat bottom without any change in phase speeds. Units are $\mathrm{mm}$ for surface elevation and $\mathrm{mm} / \mathrm{s}$ for velocity components.

period, the corresponding latitudes are $70^{\circ}, 55^{\circ}$, and $40^{\circ}$, so monthly forcing cannot generate barotropic Rossby waves poleward of these latitudes.

\section{Model and forcing}

The ocean model is an isopycnal model in spherical coordinates, and includes mixed layer physics (Jensen, 1996, 1998). It has a uniform horizontal resolution of $1 / 3^{\circ}$, covering the Indian Ocean north of $30^{\circ} \mathrm{S}$ and west of $120^{\circ} \mathrm{E}$. The model has a free surface and five layers with bottom topography confined to the deepest layer. The initial thickness is 80 , 120,250 and $600 \mathrm{~m}$ for layers $1-4$, respectively, while the thickness of layer 5 varies initially between 150 and $4950 \mathrm{~m}$, with an average of $3000 \mathrm{~m}$. The densities of layers 1-5 are 1023.6, 1025.4, 1026.5, 1027.2 and $1028.2 \mathrm{~kg} / \mathrm{m}^{3}$, respectively. The initial thickness and density for each layer was determined using temperature and salinity from the World Ocean Atlas 1994 (Levitus and Boyer, 1994; Levitus et al., 1994) as follows: the annually averaged potential density was computed in the model domain and the depths of the 1024.0, 1026.0, 1027.0 and 1027.5 isopycnals were determined. These isopycnals separate core layers in the Indian Ocean (e.g. Wyrtki, 1971, p. 219). In locations where the resulting layer thicknesses were less than $50 \mathrm{~m}$, the layers were increased by moving the interface downward and

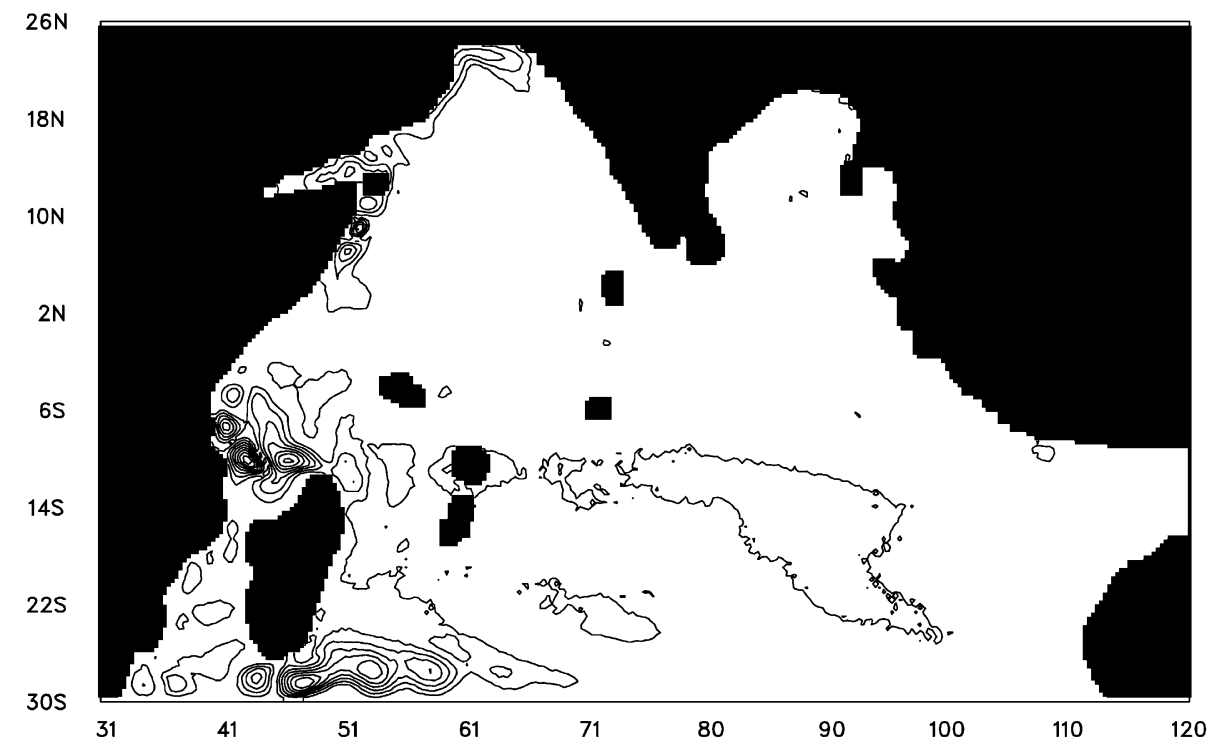

Fig. 6. Distribution of the annual mean rms error for the rescaled surface elevation $\gamma \eta$ for a GWR solution with a speed-up factor of 8 . Contour interval is $0.3 \mathrm{~cm}$. The maximum rms error is $2.6 \mathrm{~cm}$. 


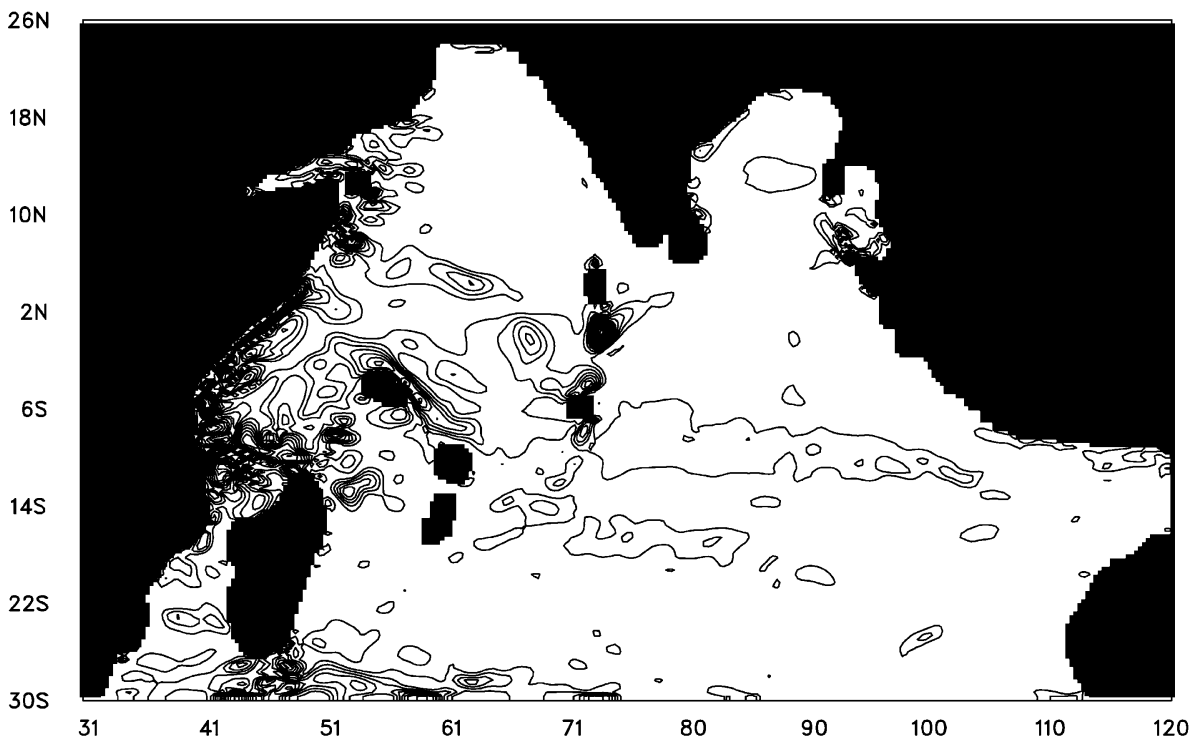

Fig. 7. Distribution of the annual mean rms error for the barotropic zonal velocity component $u$ for a GWR solution with a speed-up factor of 8 . Contour interval is $0.06 \mathrm{~cm} / \mathrm{s}$. The maximum rms error is $1.1 \mathrm{~cm} / \mathrm{s}$.

entraining fluid into the layer above. The spatial averaged depth and density for each layer were then computed and rounded to the nearest $10 \mathrm{~m}$ for layer thickness and to the nearest $0.1 \mathrm{~kg} / \mathrm{m}^{3}$ for density.
The bottom topography (National Oceanographic and Atmospheric Administration, 1986) is used without smoothing, but limited to be between 1200 and 6000 $\mathrm{m}$ (Fig. 4) in order to keep the topography in the dee-

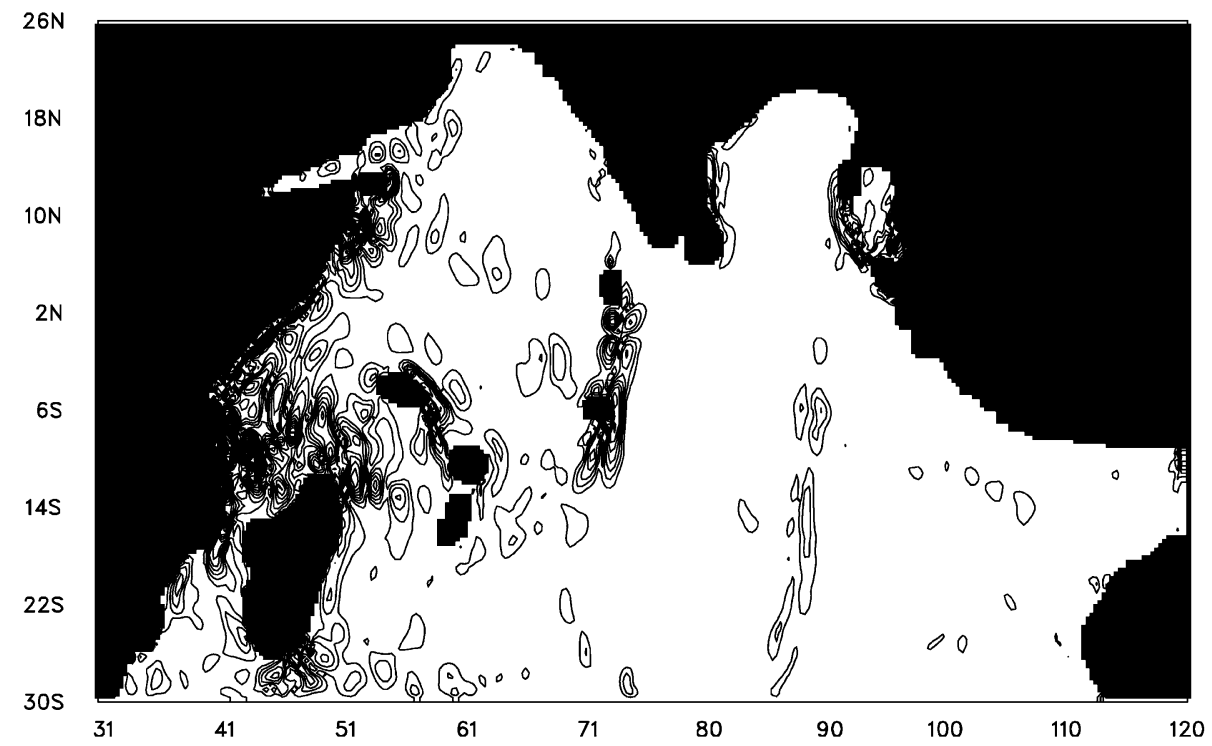

Fig. 8. Distribution of the annual mean rms error for the barotropic meridional velocity component $v$ for a GWR solution with a speed-up factor of 8 . Contour interval is $0.06 \mathrm{~cm} / \mathrm{s}$. The maximum rms error is $1.6 \mathrm{~cm} / \mathrm{s}$. 
pest layer. For simplicity, all lateral boundaries, including the southern and eastern boundaries, are closed.

Wind stress is climatological monthly mean winds from 1979 to 1988 from the European Center for Me-

\section{DAY 960}
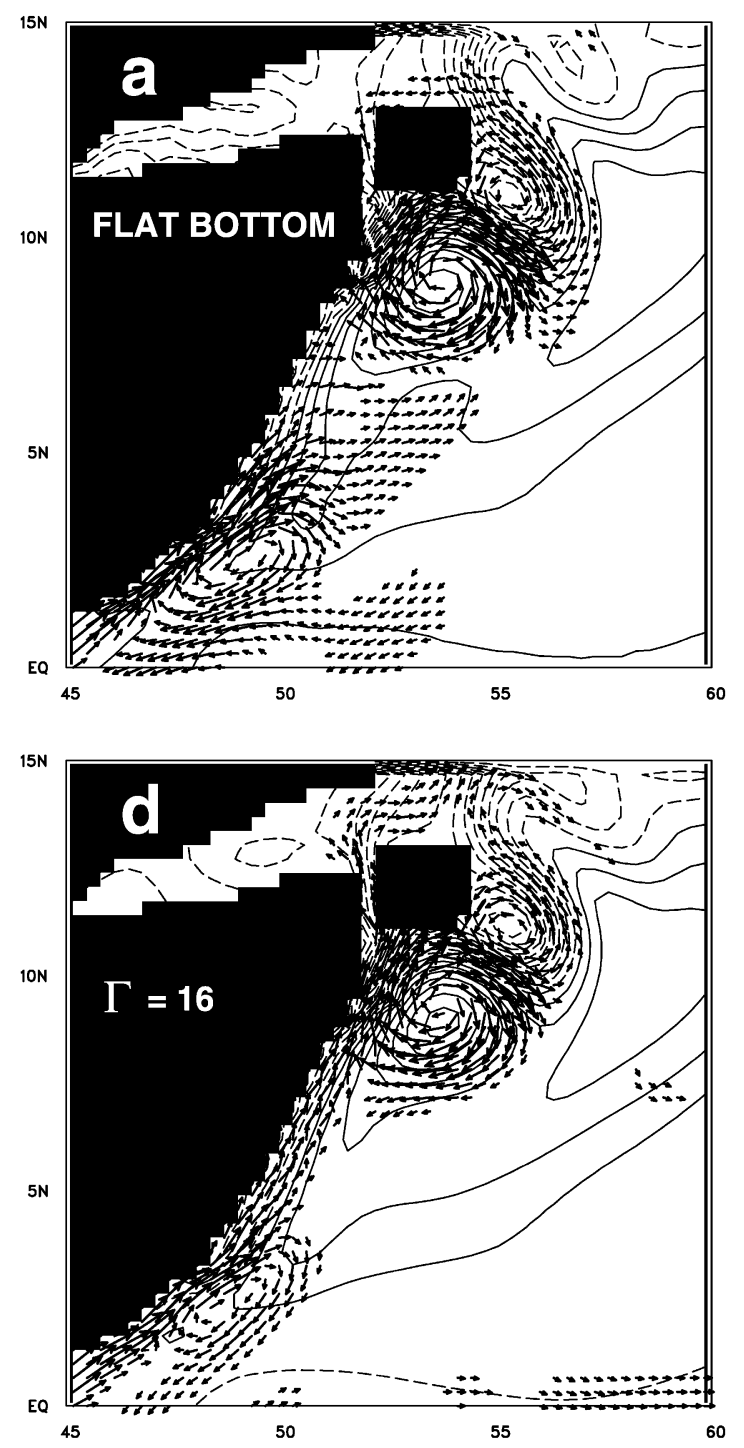

dium Range Forecast (ECMWF) reanalysis. Since the time resolution of the forcing is coarse, a linear interpolation in time is done to each model time step. Spatial interpolation was done using bi-cubic splines
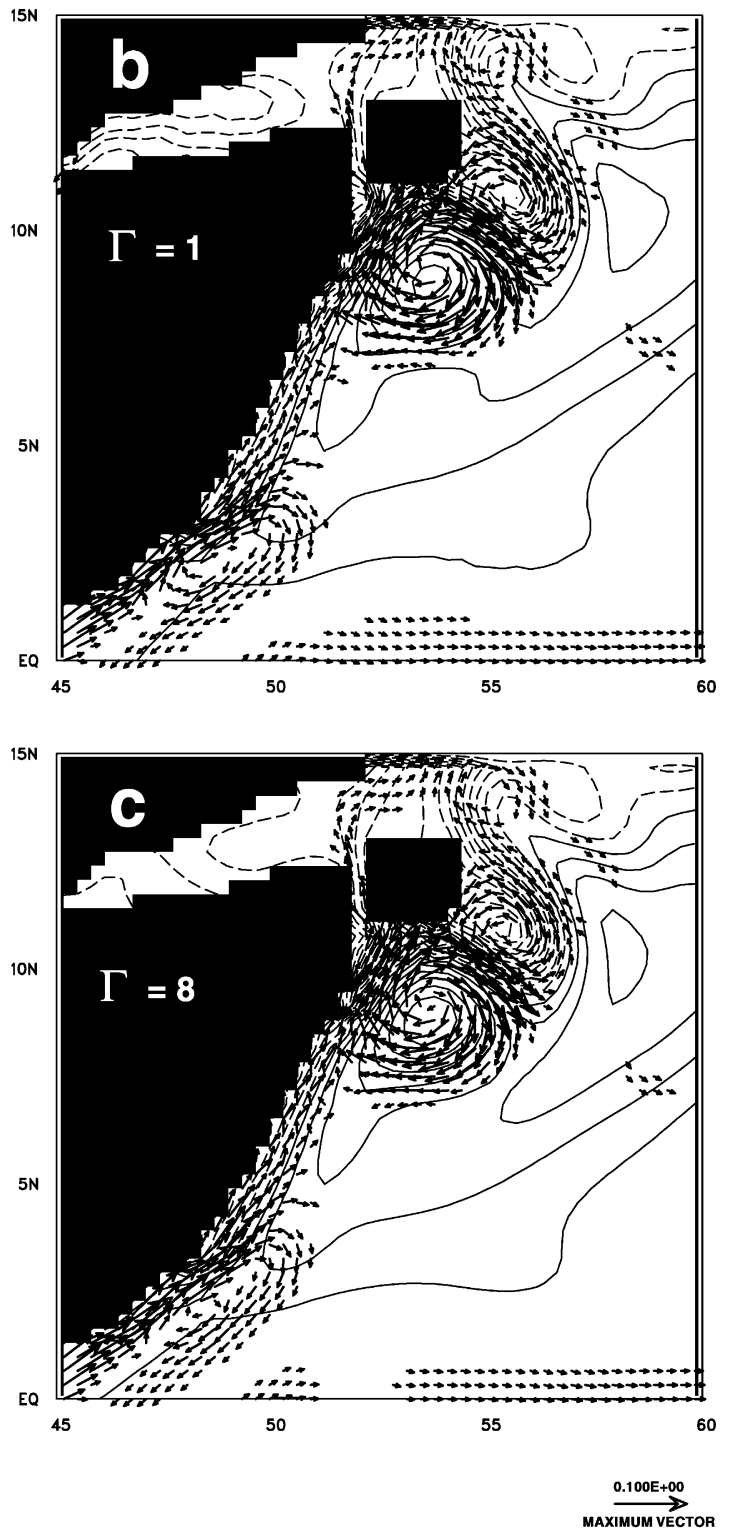

Fig. 9. Barotropic currents and rescaled surface elevation in the Somali Current region on September 15 after 960 days of integration: (a) control $(\Gamma=1)$ with flat bottom, (b) control $(\Gamma=1)$, (c) $\Gamma=8$, and (d) $\Gamma=16$. Solutions in (b) -(d) include full bottom topography. Maximum vector shown corresponds to $10 \mathrm{~cm} / \mathrm{s}$. A few longer vectors in (a) are truncated. Contour interval for the surface elevation is $2 \mathrm{~cm}$. Positive values are shown with a solid line and negative values with a dashed line. The zero contour is not shown. 
in space, which results in a continuous wind stress curl field.

Solving for Eq. (18) with the given model stratification results in the phase speeds in Table 1. Note that the phase speeds for the first mode baroclinic gravity waves are only slightly changed. Higher baroclinic modes are less affected. Also shown is the ratio of phase speeds for barotropic Rossby waves in a GWR computation to the unmodified computation for three different wavelengths.

\section{DAY 1080}
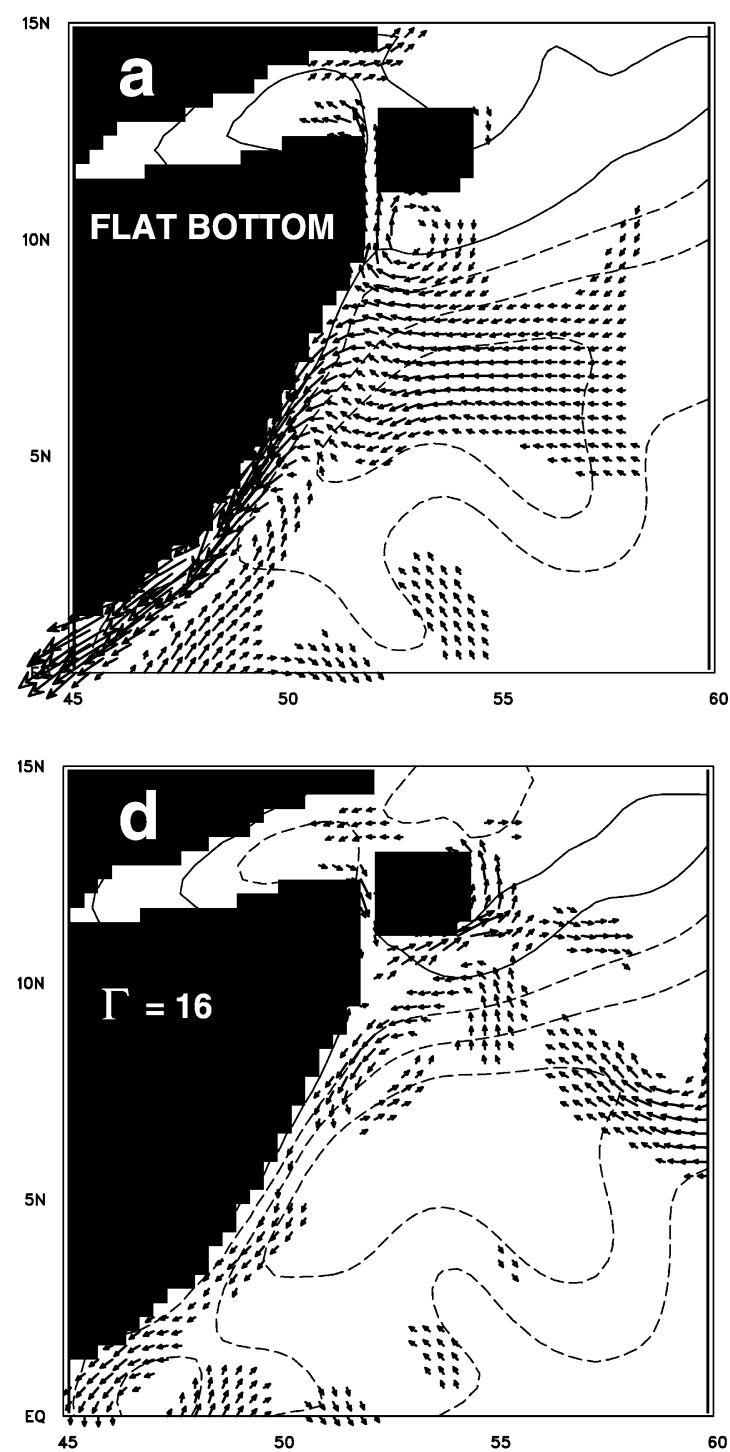
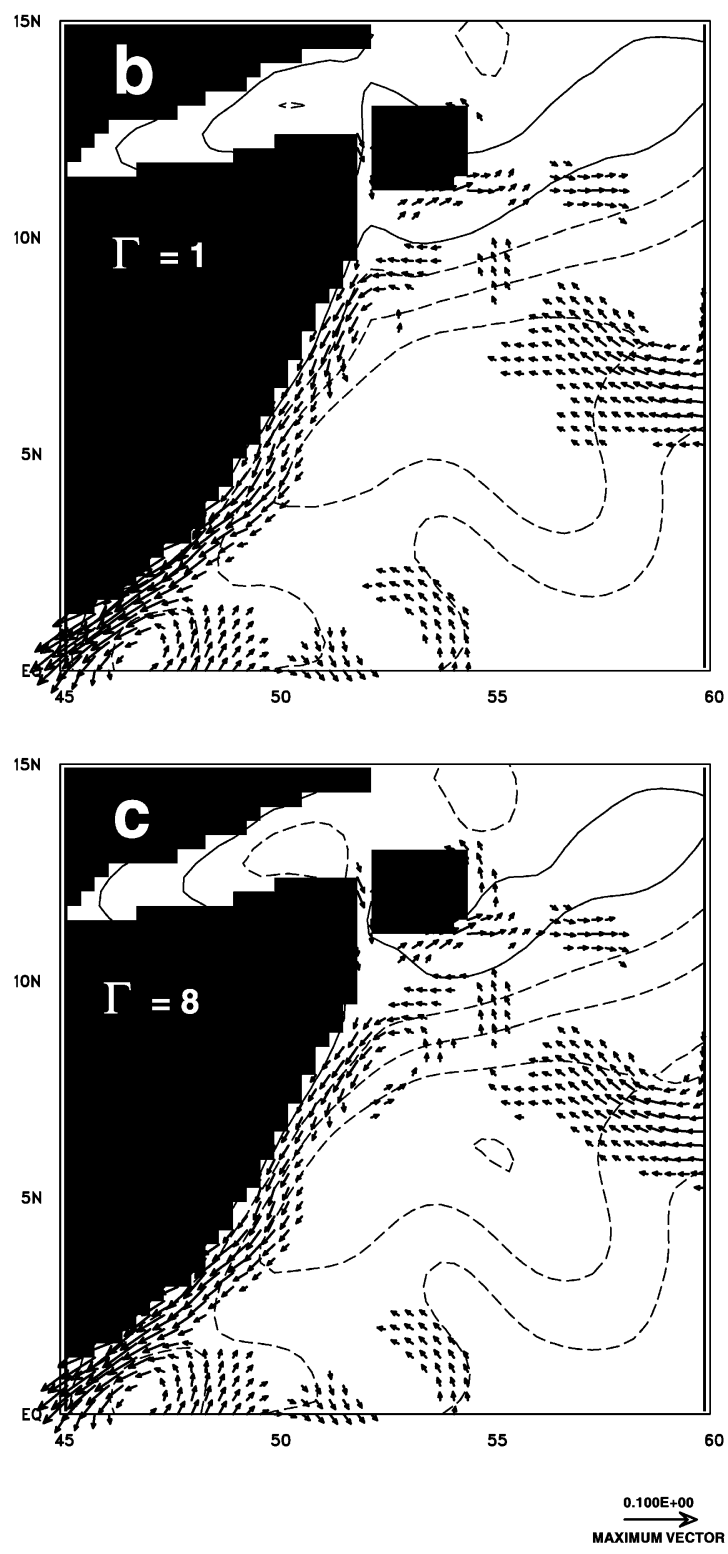

Fig. 10. As Fig. 9, but for January 15 and 1080 days of integration. 


\section{Experiments}

The circulation after 3 years of spin-up is investigated. Although fairly short, this is sufficient for computing errors related to the GWR method. The ocean model gives a realistic general circulation of the Indian Ocean as documented elsewhere (e.g. Jensen, 1991, 1993, 2001).

A control run was done without any reduction in gravity wave speed and required a time step of $45 \mathrm{~s}$. Runs with values of the speed-up factor, $\Gamma=5,8,10$, and 12 used time steps of 200,320,360, and $450 \mathrm{~s}$, respectively, while runs with $\Gamma$ values of 16 and 20 used a time step of $600 \mathrm{~s}$. In addition to these runs which include varying bottom topography, a case with a flat bottom with the same average depth of $4045 \mathrm{~m}$ and unmodified phase speeds (i.e. $\Gamma=1$ ) was computed. Since the effect of topography on the deep flow is reduced as $\Gamma$ is increased (Jensen, 2001), a minimum requirement for a meaningful GWR solution is that the errors associated with the method are less than for a flat bottom case. The flat bottom case is numerically stable for values of $\Gamma \leq 28.86$. However, in shallow areas, the barotropic gravity wave speed decreases, so a smaller value of $\Gamma$ is required to keep it larger than the baroclinic phase speed. A stable solution requires $\Gamma \leq 20$ to include areas where layer 5 may be of the order of $10 \mathrm{~m}$.

\section{Results}

No formal separation of barotropic and baroclinic flow has been made in this model calculation. For analysis, the depth-averaged flow is used as an approximation to the barotropic flow, and we will simply refer
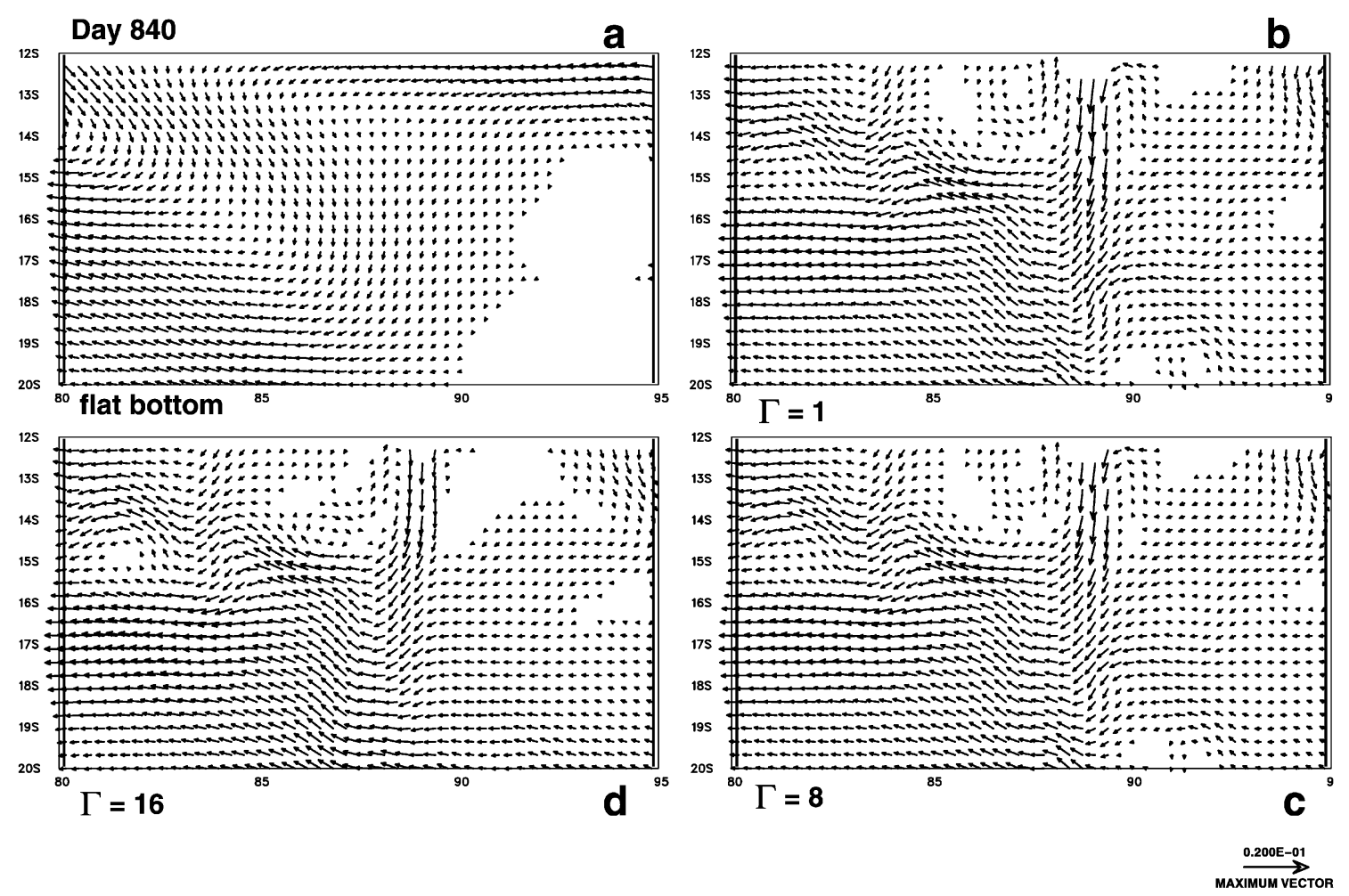

Fig. 11. Barotropic currents in a region from $80^{\circ}$ to $95^{\circ} \mathrm{E}$ and $20^{\circ}$ to $12^{\circ} \mathrm{S}$ over the Ninety East Ridge. The solution on May 15 after 840 days of integration is shown: (a) control $(\Gamma=1)$ with flat bottom, (b) control $(\Gamma=1)$, (c) $\Gamma=8$, and (d) $\Gamma=16$. Solutions in (b) - (d) include full bottom topography. Maximum vector shown corresponds to $2 \mathrm{~cm} / \mathrm{s}$. 
to it as the barotropic solution. Because of the large surface elevation associated with the GWR method, we compare solutions of $\gamma \eta$ rather than the actual surface elevation to obtain meaningful error estimates.

\section{Root mean square errors}

The root mean square (rms) errors for surface elevation and barotropic velocity components were calculated for all cases, based on differences from the control run. Fig. 5 displays the rms errors, computed over the entire model domain and averaged over a year, as a function of the speed-up parameter $\Gamma$ and for the flat bottom case.

Note that the rms errors are smaller for all GWR cases, except for $\Gamma=20$, than for the flat bottom case. This clearly means that the $\Gamma=20$ solution is unacceptable, but it also suggests that the other GWR solutions at least capture a significant part of the effect of the ocean topography. However, it does not necessarily mean that these solutions are acceptable.

The errors are not evenly distributed throughout the ocean basin. Figs. $6-8$ show the annual averaged rms error for the surface elevation $\gamma \eta$ and the two barotropic velocity components. The maximum rms error for $\gamma \eta$ is $2.6 \mathrm{~cm}$, for the zonal velocity $u, 1.1 \mathrm{~cm} /$ $\mathrm{s}$ and for the meridional velocity, $v, 1.6 \mathrm{~cm} / \mathrm{s}$. The largest errors are found on the western side of the basin in areas with frequent eddy activity and along intense currents such as western boundary currents, i.e. the Somali Current and East African Coastal Current, and the westward flowing South Equatorial Current in the region northeast of Madagascar. Away from western boundaries, the largest errors, particularly in the meridional velocity, are found over midoceanic ridges. The flow in these regions will be examined in the following sections.
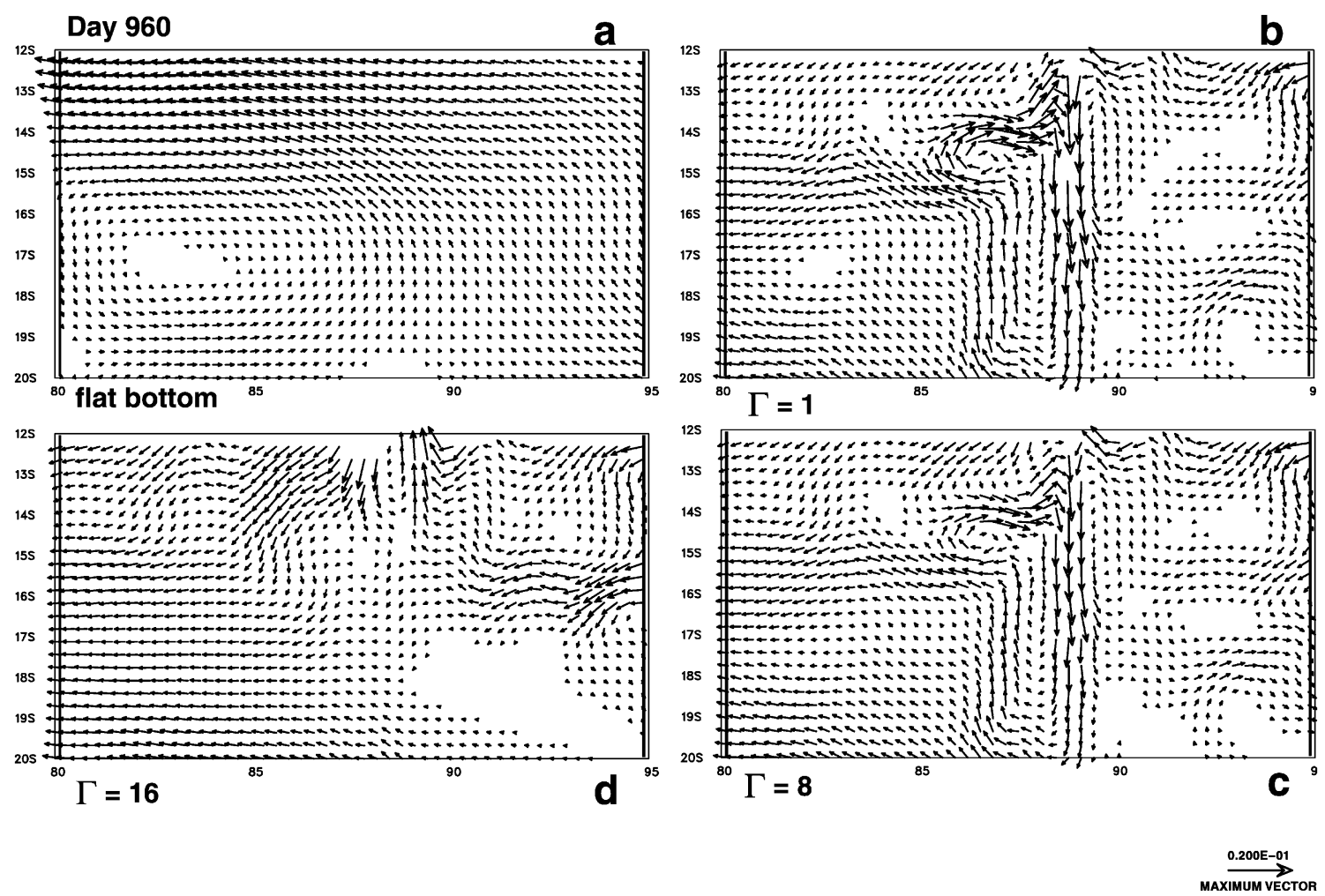

Fig. 12. As Fig. 11, but for September 15 and 960 days of integration. In (b) the maximum current reached $2.16 \mathrm{~cm} / \mathrm{s}$ at this time, but the vectors are truncated at $2 \mathrm{~cm} / \mathrm{s}$. 
It was found that the solutions for $\Gamma<8$ were in excellent agreement with the control run. The examples in the next sections will demonstrate that the $\Gamma=8$ solution is very good, while solutions using higher values of $\Gamma$ start to show significant errors. For this reason, only solutions with $\Gamma=8$ and $\Gamma=16$ will be discussed. Rather than emphasize the difference in the solutions, and thus the errors, the full solution will be shown to stress the similarities. It should also be pointed out that snapshots of the model solution are used in the next sections. For monthly averages of the solution, the errors are smaller.

\section{Western boundary currents and eddies}

The most intense current in the Indian Ocean is the annual reversing Somali Current. During the southwest monsoon, the upper ocean currents exceed $2 \mathrm{~m} / \mathrm{s}$ and associated with the current are large eddies. Fig. 9 shows solutions on September 15 after the peak of the southwest monsoon. The large eddy centered at $53^{\circ} \mathrm{E}$ and $9^{\circ} \mathrm{N}$ is the Great Whirl found during the boreal summer from June through October. It forms just north of the equator in June, but migrates northward during the southwest monsoon. All solutions, including the two GWR solutions $(\Gamma=8$ and $\Gamma=16)$, are very good. However, due to small phase errors in connection with movement of the eddy and the strong currents, the rms error becomes large. North of the island of Socotra, the GWR solutions with bottom topography are in better agreement with the control, and along the equator, a weak eastward flow is missing from the flat bottom solution.

During the northeast monsoon season the flow is southward in the boundary current (Fig. 10). The major difference between the solutions is that the Somali Current is fed by a $5^{\circ}$-wide eastward zonal flow in the flat bottom case, while in the cases with bottom topography, onshore flow follows the bottom topography along the Carlsberg Ridge and feeds the Somali Current in a narrow band at $10^{\circ} \mathrm{N}$. The GWR
Day 1080

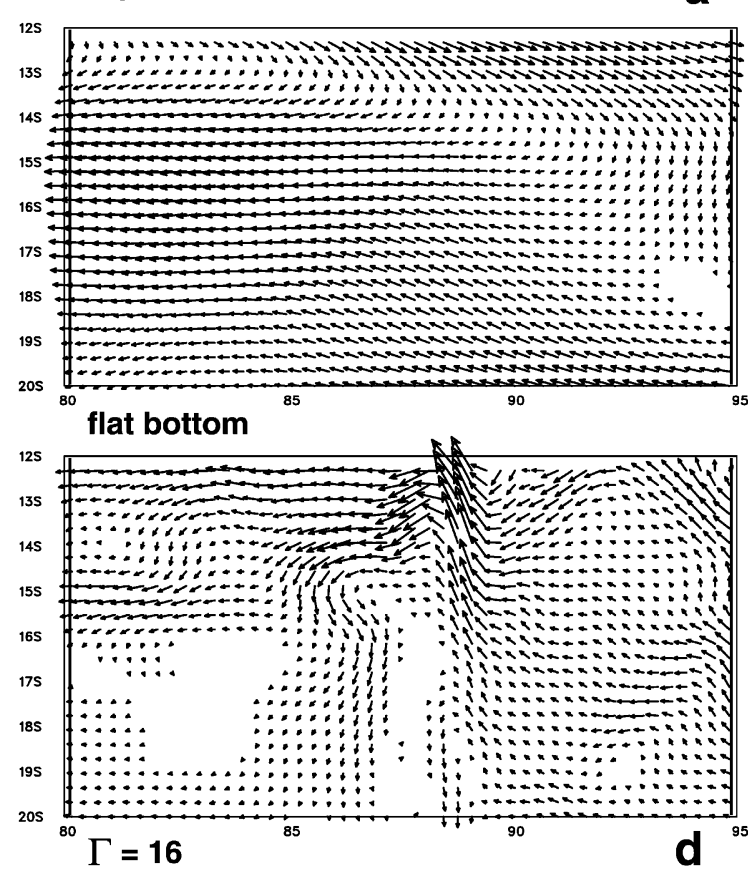

a

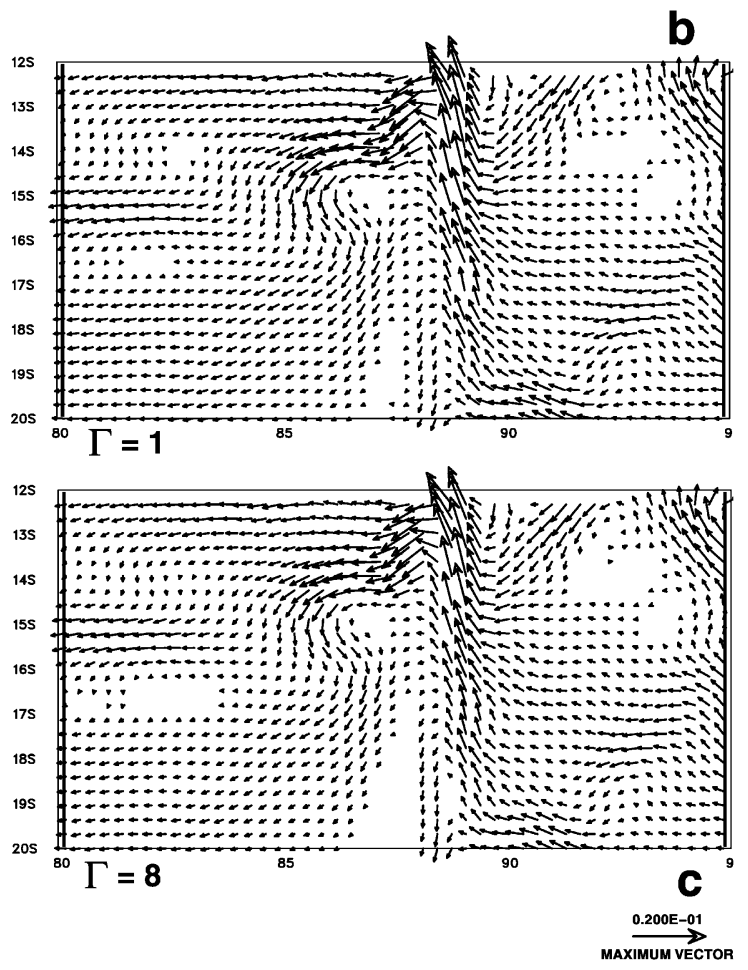

Fig. 13. As Fig. 11, but for January 15 and 1080 days of integration. 
solutions include this feature, but the solution using $\Gamma=16$ has a boundary current that is much weaker than in the control run.

\section{Oceanic ridges}

Figs. 10-13 show solutions on May 15 , September 15 and January 15 , respectively, in a region $80^{\circ}-95^{\circ} \mathrm{E}$ and $20^{\circ}-12^{\circ} \mathrm{S}$ over the shallowest part of the Ninety East Ridge. The depth rises from 5000 to $2400 \mathrm{~m}$ over $200 \mathrm{~km}$ and drops to the same depth again over about the same distance. The solution with a flat bottom is, not surprisingly, very different from the other solutions, but shows more clearly the passage of the windforced, annual barotropic Rossby wave with a meridional wavelength of about $650 \mathrm{~km}$. This annual wave causes a strong, trapped circulation around the peak of the seamount in the solutions with bottom topography. Using a speed-up factor of 16 , the solution is acceptable during a large part of the year, as illustrated by solutions at day 840 (Fig. 11) and day 1080 (Fig. 13), but clearly inadequate around day 960 (Fig. 12).

\section{Arabian sea}

Rossby waves are radiated from the west coast of India and propagate across the Arabian Sea (e.g. Jensen, 1991). Although the oceanic response is mainly baroclinic in nature, the shift in the wind stress at the onset of each monsoon season will, in addition, generate weak barotropic waves. The Arabian Sea is also the area in the model with the largest distance from the equator and not influenced by an artificial boundary, and was for these two reasons chosen as a third example of GWR solution performance. Fig. 14 shows the flow in June just after the onset of the southwest monsoon. The flat bottom case is dominated by northward barotropic flow, a fast response to winds from the southwest. In the cases with bottom topography, the barotropic response is delayed and
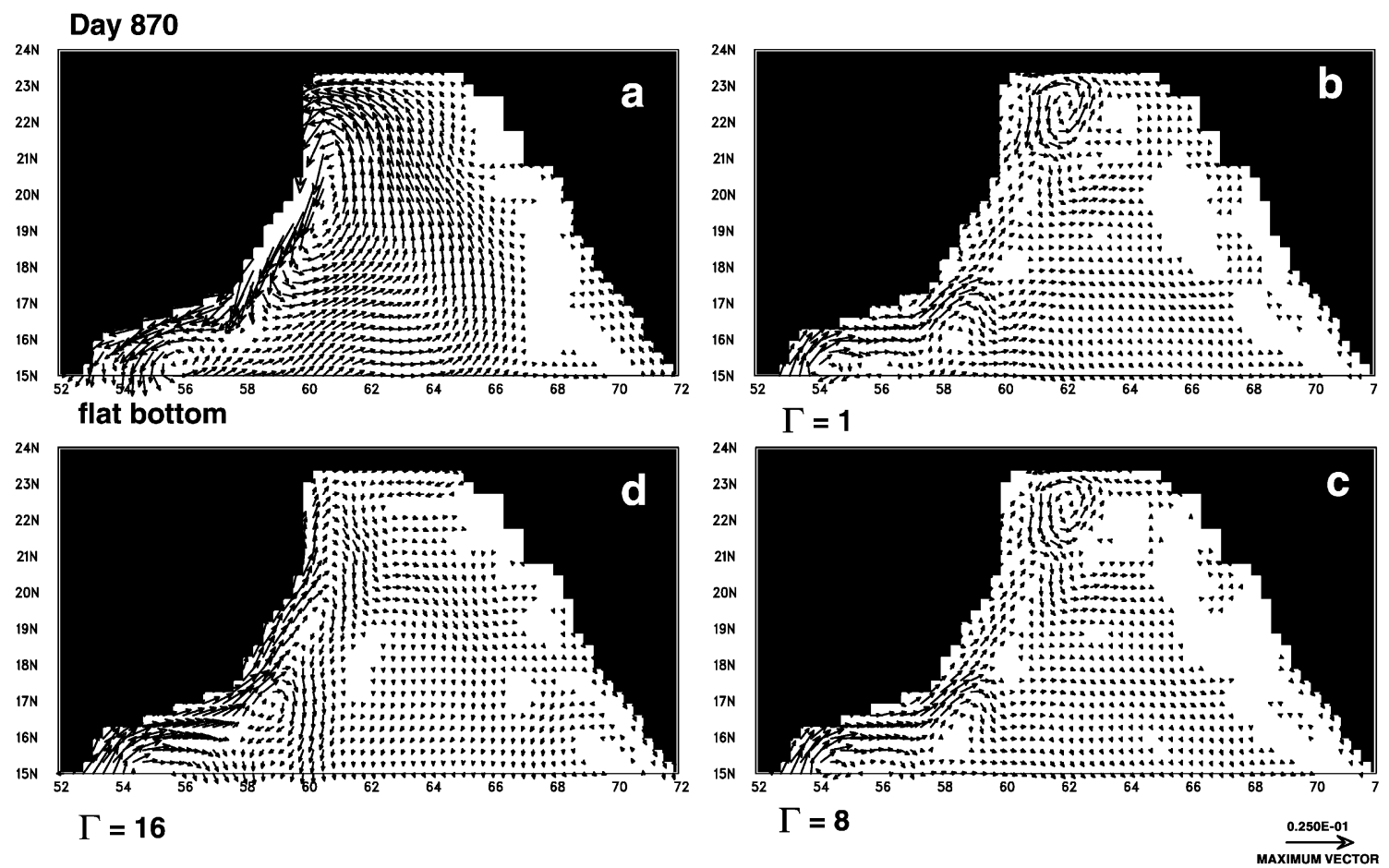

Fig. 14. Barotropic currents in the Arabian Sea north of $15^{\circ} \mathrm{N}$. The solution on June 15 after 870 days of integration is shown: (a) control $(\Gamma=1)$ with flat bottom, (b) control ( $\Gamma=1)$, (c) $\Gamma=8$, and (d) $\Gamma=16$. Solutions in (b) - (d) include full bottom topography. Maximum vector shown corresponds to $2.5 \mathrm{~cm} / \mathrm{s}$. For the flat bottom case, longer vectors are truncated and some vectors have been removed for clarity. 
strongly modified. It should be mentioned that the total flow in the upper ocean is almost the same with and without bottom topography, since the response to the wind primarily is baroclinic. The $\Gamma=16$ solution is quite good, but has a relatively strong southward flow at $60^{\circ} \mathrm{E}$ and the cyclonic eddy by the Persian Gulf is missing.

At the onset of the northeast monsoon season (Fig. 15), the flat bottom case shows a similarly strong response, this time with reversed flow. With bottom topography, the series of eddies along the Arabian coast are all fairly well represented by the GWR solutions, although some significant differences between the control and the $\Gamma=16$ case can be seen. For instance, along the Arabian coast, from $18^{\circ}$ to $20^{\circ} \mathrm{N}$, a southward coastal current and an offshore counter current are found in the $\Gamma=16$ solution, but are not seen in the control or the $\Gamma=8$ solutions. During other times of the year, the GWR solutions have less error than in the examples given here.

\section{Longer integrations and global models}

An Oceanic General Circulation Model for a global domain without a special numerical treatment of the barotropic mode has yet to be run for a simulation of several years or longer. Such a computation would require too much computer time to be practical. This implies that we are assuming that errors introduced by time-splitting, rigid lids, semiimplicit methods or other numerical techniques are acceptable, although we do not have error estimates.

The model used in this study was designed to be a regional, eddy-resolving model, and the results shown above are for 3-year integrations and a limited geographical area. The integration time was chosen to be sufficiently long to allow the first baroclinic Rossby wave to propagate across the ocean and establish a circulation in balance with the wind stress, and the limited domain ensured that a control run with $\Gamma=1$ was computationally feasible.

\section{Day 1050}
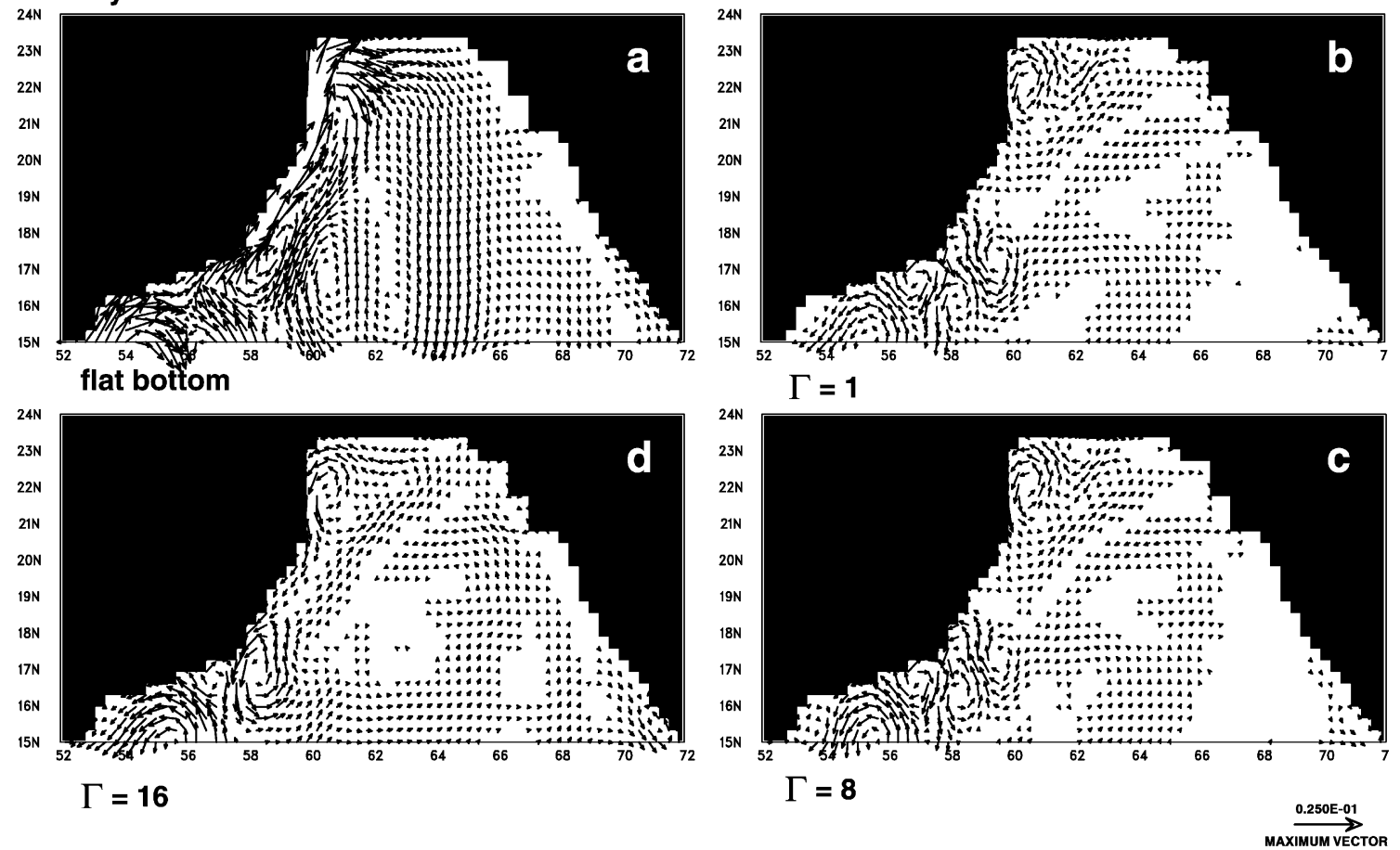

Fig. 15. As Fig. 14, but for December 15 and 1050 days of integration. 
As the ocean circulation is spun up further, an accumulation of errors due to numerical differences between the control run and the GWR run can be expected. For instance, while the time truncation error is smaller in the control run, the numerical viscosity introduced by the time filter is larger than for the GWR runs. By extending the control run and the $\Gamma=8$ run to 12 years, the rms errors were found to have increased by about $65 \%$ for the scaled surface elevation $\gamma \eta$ and for the zonal velocity component, while the rms error for the meridional velocity increased by a factor of 2. The increase in rms errors continued during years 3 to 12 , but with a decreasing rate. Overall, the barotropic currents (panels $\mathrm{b}$ and $\mathrm{c}$ in Figs. 9-15) showed little change between year 3 and year 12 at any day throughout the year.

The model was also extended to include a nearglobal domain, covering the globe from $60^{\circ} \mathrm{S}$ to $60^{\circ} \mathrm{N}$. Periodic boundary conditions were used in the zonal direction, and the resolution was kept at $1 / 3^{\circ}$. The model was run for 3 years using $\Gamma=8$ and $\Gamma=16$. The differences between the two runs were used to estimate rms errors for tropical areas outside the Indian Ocean. In the tropical region $\left(20^{\circ} \mathrm{S}\right.$ to $\left.20^{\circ} \mathrm{N}\right)$, the rms errors for the surface elevation and the meridional velocity were slightly higher in the Indian Ocean than the average over all tropical oceans. The rms error for the zonal velocity was $33 \%$ smaller in the Indian Ocean. The largest errors were found in the Somali Current and the New Guinea Coastal Current. Overall, it seems that the error estimation in the Indian Ocean represent the errors in the tropical oceans quite well.

Table 1

Barotropic and first mode baroclinic gravity wave phase speed $(\mathrm{m} / \mathrm{s})$ and fraction of correct Rossby wave phase speed for three wavelengths

\begin{tabular}{lcllll}
\hline $\begin{array}{l}\text { Speed-up } \\
\text { factor }(\Gamma)\end{array}$ & $\begin{array}{l}\text { External } \\
\text { gravity }\end{array}$ & $\begin{array}{l}\text { First } \\
\text { internal }\end{array}$ & \multicolumn{3}{l}{ Wavelength } \\
\cline { 4 - 6 } & & & $500 \mathrm{~km}$ & $1000 \mathrm{~km}$ & $1500 \mathrm{~km}$ \\
\hline 1 & 199.2 & 3.11 & 1.00 & 1.00 & 1.00 \\
3 & 66.3 & 3.12 & 0.99 & 0.95 & 0.90 \\
4 & 47.7 & 3.12 & 0.98 & 0.91 & 0.82 \\
5 & 39.7 & 3.13 & 0.96 & 0.86 & 0.75 \\
8 & 24.6 & 3.15 & 0.91 & 0.72 & 0.53 \\
10 & 19.6 & 3.18 & 0.86 & 0.61 & 0.41 \\
12 & 16.2 & 3.21 & 0.82 & 0.52 & 0.33 \\
16 & 11.8 & 3.29 & 0.71 & 0.38 & 0.22 \\
20 & 9.1 & 3.42 & 0.61 & 0.28 & 0.17 \\
\hline
\end{tabular}

Outside the tropics, the baroclinic circulation is far from equilibrium, so a quantitative error estimation is not meaningful. However, regions where errors are large can be identified, and they are likely to remain areas with relative large errors for longer integrations. In the subtropics, the largest differences are found in the western boundary currents, e.g. the Algulhas Current, Brazil Current and in the eddy fields off the west coast of South Africa and South America. In mid-latitudes, the Kuroshio, the Gulf Stream and the Malvinas Current have the largest errors.

There are several reasons for increased errors in the extended domain. From Eq. (24), we found that Rossby waves with bi-monthly period only can exist equatorward of $40^{\circ}$ for $\Gamma=8$, and from Table 1, we see that the longest barotropic Rossby waves are significantly slowed down as $\Gamma$ increases. This raises the potential for particularly large errors in the Southern Ocean. In the short near-global integration done here, the largest errors were indeed found in that ocean.

\section{Summary and discussion}

The gravity wave retardation (GWR) method was introduced as a potential alternative way to solve for the barotropic mode. It was shown that the method essentially corresponds to a physical system where the air is replaced with a fluid of much higher density. For instance, if the GWR speed-up factor is 8 , the density of the fluid is $98.4 \%$ of the sea water. The associated large deviations in surface elevation in the modified solution may seem severe, and do introduce errors. However, for the purpose of sea level analysis, for instance a comparison with satellite observations, the surface displacement in the GWR solution can be rescaled by multiplication of the GWR parameter $\gamma$ to give very good results.

The errors introduced by the GWR method for barotropic Rossby waves are most severe for waves with scales of the order of the external Rossby radius of deformation or larger. Fortunately, the scale of weather systems which force the ocean is smaller, which makes it possible to use the method for realistic forcing.

It was demonstrated using a five-layer model with bottom topography and climatological wind forcing, 
that the GWR method can produce realistic solutions. For solutions with speed-up factors up to 16 , it was found that the rms errors for rescaled sea level and for barotropic current components were less than for a flat bottom case without wave distortion. Errors were largest along the western side of the basin and along oceanic ridges and areas of similarly steep bottom topography.

Solutions for the barotropic mode are remarkably good for speed-up factors of about 8 for the case of monthly forcing in a tropical ocean. For high-frequency wind forcing, i.e. less than 30 days period, the oceanic response in mid-latitudes is essentially purely barotropic, but very weak compared to the response to seasonal forcing. In order to model the resulting flow adequately, Tobis (1996) found that the speed-up factor should not exceed 4. However, these high-frequency barotropic flows have not been demonstrated to be of importance for climate simulations.

When the GWR speed-up factor is increased, the error increases, but the same is true for implicit methods as the time step is increased to speed-up calculations. The major advantage of the GWR method compared to other methods is its easy implementation and explicit time step that allows straightforward parallelization of the code. For a layer model, the method does not require a separation of the barotropic mode, and can be implemented by minor changes to the code. For an OGCM with time splitting, a single parameter can substantially reduce the computational cost. The method can be used to save significant computational resources for model testing, for parameter studies, or for simulations of tropical oceans where high accuracy of the barotropic mode may not be needed. However, as the vertical resolution of the model increases, the relative cost of the barotropic calculation decreases, and the benefit of the GWR method will be reduced.

\section{Acknowledgements}

This research was funded by Frontier Research System for Climate Change. School of Ocean and Earth Science and Technology Contribution Number 6036, and International Pacific Research Center Contribution Number IPRC-166.

\section{References}

Bleck, R., Smith, L., 1990. A wind-driven isopycnic coordinate model of the North and Equatorial Atlantic Ocean: Part I. Model development and supporting experiments. J. Geophys. Res. 95, 3273-3286.

Blumberg, A.F., Mellor, G.L., 1987. A description of a three-dimensional coastal ocean circulation model. In: Heaps, N. (Ed.), Three-dimensional Coastal Ocean Models. American Geophysical Union, Washington, DC, pp. 1-16.

Bryan, K., 1969. A numerical method for the study of the circulation of the world ocean. J. Comput. Phys. 4, 347-376.

Bryan, K., 1984. Accelerating the convergence to equilibrium of ocean-climate models. J. Phys. Oceanogr. 14, 666-673.

Chorin, A.J., 1967. A numerical method for solving incompressible viscous flow problems. J. Comput. Phys. 2, 12-26.

Dukowicz, J.K., Smith, R.D., 1994. Implicit free-surface method for the Bryan-Cox-Semtner ocean model. J. Geophys. Res. 99, $7991-8014$.

Gill, A.E., 1982. Atmosphere-Ocean Dynamics. Academic Press, Orlando, $662 \mathrm{pp}$.

Hallberg, R., 1997. Stable split time stepping schemes for large scale ocean modelling. J. Comput. Phys. 135, 54-65.

Hearn, C.J., Hunter, J.R., 1987. Modelling wind-driven flow in shallow water systems on the southwest Australian coast. In: Noye, J. (Ed.), Numerical Modelling: Applications to Marine Systems. Elsevier (North-Holland), pp. 47-57.

Hunter, J.R., 1990a. User manual for numerical hydrodynamic models of marine systems and associated plotting package. CSIRO Division of Oceanography. Report OMR-14/00, 73 pp.

Hunter, J.R., 1990b. User manual for three-dimensional numerical hydrodynamic and dispersion model. CSIRO Division of Oceanography. Report OMR-24/00, 44 pp.

Jensen, T.G., 1991. Modeling the seasonal undercurrents in the Somali current system. J. Geophys. Res. 96, 22151-22167.

Jensen, T.G., 1993. Equatorial variability and resonance in a winddriven Indian Ocean model. J. Geophys. Res. 98, 22533-22552.

Jensen, T.G., 1996. Artificial retardation of barotropic waves in layered ocean models. Mon. Weather Rev. 124, 1272-1283.

Jensen, T.G., 1998. Description of a Thermodynamic Ocean Modelling System (TOMS). Dept. of Atmospheric Science Paper No. 670, Colorado State University, 50 pp.

Jensen, T.G., 2001. Application of the GWR method to the tropical Indian Ocean. Mon. Weather Rev. 129, 470-485.

Killworth, P.D., Stainforth, D., Webb, D.J., Paterson, S.M., 1991. The development of a free-surface Bryan-Cox-Semtner ocean model. J. Phys. Oceanogr. 21, 1333-1348.

Levitus, S., Boyer, T.P., 1994. Temperature, NOAA Atlas NESDIS 4. World Ocean Atlas 1994, vol. 4. U.S. Government Printing Office, Washington, DC. 117 pp.

Levitus, S., Burgett, R., Boyer, T.P., 1994. Salinity, NOAA Atlas NESDIS 3. World Ocean Atlas 1994, vol. 5. U.S. Government Printing Office, Washington, DC. 99 pp.

National Oceanographic and Atmospheric Administration, 1986. ETOP05 digital relief of the surface of the Earth. It Data Announce, 86-MGG-07, Nat. Geophys. Data Center, Washington, DC. 
Oberhuber, J.M., 1993. Simulation of the Atlantic circulation with a coupled sea ice-mixed layer-isopycnal general circulation model: Part I. Model description. J. Phys. Oceanogr. 23, $808-829$.

Semtner, A.J., 1986. Finite-difference formulation of a world ocean model. In: O'Brien, J.J. (Ed.), Advanced Physical Oceanographic Numerical Modelling. Reidel, Boston, pp. 187-202.
Tobis, M., 1996. Effects of slowed barotropic dynamics in parallel ocean climate models. PhD Dissertation, January 1996, Univ. of Wisconsin, Madison.

Wyrtki, K., 1971. Oceanographic Atlas of the International Indian Ocean Expedition. National Science Foundation, Washington, DC. $531 \mathrm{pp}$. 